\title{
Original Artical
}

\section{Analysis of Pain Assessment and Nursing Measures for Patients with Advanced Hematological Tumors}

\author{
Min Shao* \\ Tangdu Hospital, the Second Affiliated Hospital of Air Force Military Medical University, Shaanxi 710000, China \\ Corresponding author: Tangdu Hospital, the Second Affiliated Hospital of Air Force Military Medical \\ University, Shaanxi 710000, China. E-mail: 349179920@qq.com
}

\section{Abstract}

Objective: To analyze the pain assessment results and nursing measures and its effects on patients with advanced hematological tumors. Methods: Select 50 patients with advanced hematological tumors admitted to our hospital from January 2017 to January 2019, evaluating their pain levels when implementing pain care. Results: Among 50 patients with advanced hematological tumors, mild pain was $6.00 \%$, moderate pain was $50.00 \%$, and severe pain was $44.00 \%$. The quality of life scores was increasing while the pain level assessment, Pittsburgh sleep quality index and mental state scores decreased after the implementation of pain care. The difference was statistically significant $(P<0.05)$. Conclusion: Moderate and severe pain is common in patients with advanced hematological tumor. Considerate pain care is helpful to relieve pain, improve sleep quality and mental state.

Keywords

Advanced hematological tumor patients; pain assessment; pain nursing

\section{INTRODUCTION}

Hematological tumor has become one of the fastest growing tumor with high incidence rate in all malignant tumors in China. It contains three major categories: namely leukemia, malignant lymphoma and multiple myeloma, among which leukemia and lymphoma are relatively common ${ }^{[1]}$. With the prolongation of the course of disease, the aggravation of the disease, and the long-term treatment of radiotherapy and chemotherapy, patients with terminal hematological tumor generally have different degrees of pain. Therefore, it not only leads to the serious psychological problems, but also weakens their sleep quality. Ulteriorly it will reduce the body quality, and then their tolerance to clinical treatment is greatly reduced. Thus, it has become an urgent task to give them

(i) (c) The Author(s) 2020. Open Access This article is licensed under a Creative Commons Attribution 4.0 International License (https://creativecommons.org/licenses/by/4.0/), which permits unrestricted use, sharing, adaptation, distribution and reproduction in any medium or format, for any purpose, even commercially, as long as you give appropriate credit to the original author(s) and the source, provide a link to the Creative Commons license, and indicate if changes were made. 
well pain care ${ }^{[2]}$. So this study focused on 50 cases of patients with advanced hematological tumors treated from January 2017 to January 2019 in our hospital to carry out pain assessment and implementation of pain care, which has achieved relatively ideal results. It was summarized as follows.

\section{DATA AND METHODS}

\subsection{General data:}

After the approval of the medical ethics committee, 50 cases of patients with advanced hematological tumors in our hospital treated from January 2017 to January 2019 were selected, among them 22 are males and 28 are females. Their average age was $(42.10 \pm 1.10)$ years old. And there are 25 cases of leukemia ( 15 cases of acute non lymphocytic leukemia, 10 cases of acute lymphocytic leukemia), 15 cases of non Hodgkin's lymphoid of tumor and 10 cases of multiple myeloma.

\section{a. Inclusion criteria:}

1) all patients are diagnosed as hematological tumors and are in advanced stage.

2) They have good cognitive function and can cooperate with pain assessors.

3) They agree with the study protocol.

b. Exclusion criteria:

1) Participants in other clinical trials.

2) Patients with brain organic diseases.

3) Cognitive or mental disorders.

\subsection{Methods}

The trained doctors used visual analog scoring method to evaluate the pain degree of patients. The specific methods are as follows: draw a line of $10 \mathrm{~cm}$ on the surface of A4 paper with a ruler, and make a scale at $1 \mathrm{~cm}$, where $0 \mathrm{~cm}$ represents no pain and $10 \mathrm{~cm}$ represents unbearable severe pain ${ }^{[3]}$. After drawing, the doctor will explain the specific integration method and the value of each scale to all patients with advanced hematological tumor. And then the patients should select the most appropriate scale according to their own feelings. After all patients were depicted, the results were sorted out and analyzed. All patients with advanced hematological tumor were given pain nursing, and the specific plan was as follows:

\section{a. Analgesic care:}

Obeying the World Health Organization's rules for pain relief, non opioid drugs are used for mild pain relief, including ibuprofen sustained release capsules / ibuprofen controlled release tablets, paracetamol tablets, etc.; moderate pain patients are given weak opioid analgesics, such as paracetamol dihydrocodeine tablets, tramadol hydrochloride tablets, bucinazine hydrochloride tablets, and paracetamol codeine tablets; for severe pain patients, strong opioid drugs are used opioids and non opioid analgesics.Strong opioid drugs include morphine sulfate controlled-release tablets, oxycodone hydrochloride controlled-release tablets, etc $^{[4]}$. During the period of taking analgesic drugs, the patients are closely observed for adverse reactions. The doctors should evaluate the analgesic effect scientifically. And the dosage of analgesic drugs is dynamically adjusted accordingly. Because opioids are addictive, we should explain to the patients and their families before administration, and start from small doses to avoid drug dependence caused by large doses.

\section{b. Non drug pain relief:}

Play soft and soothing, light and lively, or deep and thick music in the ward, or master the correct rhythmic deep breathing skills under the personal demonstration of medical staff, in order to further reduce the pain feeling of their body. The patients with activity ability can carry out moderate aerobic exercise in the company of nursing staff or family members to improve the body quality and pain tolerance threshold.

\section{c. Psychological nursing actively:}

Actively communicate with patients, and patiently listen to the heartfelt wishes of patients, and express 
their approval and support for the content of their expression. Encourage them to recall the past happy emotional experience and analyze the causes of pain together, so as to change the situation and win the full trust of patients. Care for patients with a high degree of compassion and sense of responsibility, and give detailed answers to their questions. Each communication should not talk about too much, but should adopt the method of grading to make them really master. Because the patients with advanced blood tumor have lost the chance of cure, it is helpful to reduce the pain by reducing their psychological trauma. When the side effects of treatment occur, we should comfort and encourage them, and reduce their psychological pressure by talking many times.

\subsection{Observation index}

The pain assessment results, pain score, Pittsburgh sleep quality index, quality of life score and psychological state score were taken as the observation indexes. The score of pain was measured by visual analogue scale. The total score was $0-10$. The higher the score, the more severe the pain. Pittsburgh sleep quality index was measured by Pittsburgh sleep quality index scale. The total score was 0-21 points. The higher the score, the worse the sleep quality. The quality of life score was evaluated by the quality of life score of tumor patients. The total score was 0-60 points. The higher the score, the higher the quality of their life. The score of psychological state was composed of anxiety score and depression score. Anxiety score was measured by self rating anxiety scale. The Chinese norm boundary value was 53 points. The higher the value was, the more serious anxiety was; the depression score was measured by self rating depression scale. The Chinese norm boundary value was 50 points. The higher the value was, the more serious the depression was.

\subsection{Statistical methods:}

Spss22.0 statistical software was used for processing. The measurement data were expressed by mean \pm standard deviation $(\bar{x} \pm \mathrm{s})$ and $t$-test. The adoption rate of counting data (\%) was expressed. The difference was statistically significant $(P<0.05)$.

\section{RESULTS}

\subsection{Pain assessment results}

The 50 patients with advanced hematological tumors in this study generally have moderate to severe pain, including mild pain $6.00 \%$, moderate pain $50.00 \%$, and severe pain $44.00 \%$. The specific evaluation results are shown in Table 1.

Table 1 Pain assessment results $[n(\%)]$

\begin{tabular}{cccccc}
\hline Type of disease & $n$ & No pain & Mild pain & Moderate pain & Severe pain \\
\hline $\begin{array}{c}\text { Acute } \\
\text { nonlymphocytic } \\
\text { leukemia } \\
\text { Acute } \\
\text { lymphocytic } \\
\text { leukemia }\end{array}$ & 15 & $0(0.00)$ & $1(6.67)$ & $7(46.67)$ & $7(46.67)$ \\
Non-Hodgkin's Lymphoma & 10 & $0(0.00)$ & $1(10.00)$ & $5(50.00)$ & $4(40.00)$ \\
Multiple myeloma & 15 & $0(0.00)$ & $1(6.67)$ & $8(53.33)$ & $6(40.00)$ \\
In total & 10 & $0(0.00)$ & $0(0.00)$ & $5(50.00)$ & $5(50.00)$ \\
\hline
\end{tabular}

\subsection{Comparison of pain scores, sleep quality and quality of life scores before and after pain nursing}

The quality of life scores was increasing while the pain level assessment, Pittsburgh sleep quality index and mental state scores decreased after the implementation of pain care. The difference was statistically significant $(P$ 
$<0.05)$. It can be seen in Table 2 .

Table 2 Comparison of pain scores, sleep quality scores, life quality scores before and after pain nursing ( $\bar{x}$ \pm s,score)

\begin{tabular}{cccc}
\hline Period & Pain score & Pittsburgh sleep quality index & Quality of life scores \\
\hline Before $(n=50)$ & $7.85 \pm 0.35$ & $17.50 \pm 0.50$ & $14.50 \pm 1.50$ \\
After $(n=50)$ & $1.40 \pm 0.20$ & $7.00 \pm 1.00$ & $40.00 \pm 1.00$ \\
$t$ & 9.987 & 11.242 & 59.680 \\
$P$ & 0.000 & 0.000 & 0.000 \\
\hline
\end{tabular}

\subsection{Comparison of mental status scores before and after pain nursing}

The score of mental state after pain nursing was lower than before, the difference was statistically significant $(P$ $<0.05)$, seen in Table 3 .

Table 3 Comparison of mental status scores before and after pain nursing $(\overline{\boldsymbol{x}} \pm \mathbf{s}$,score $)$

\begin{tabular}{ccc}
\hline Period & Anxiety score & Depression score \\
\hline Before $(n=50)$ & $71.00 \pm 1.00$ & $70.50 \pm 1.50$ \\
$\operatorname{After}(n=50)$ & $52.50 \pm 1.50$ & $50.00 \pm 1.00$ \\
$t$ & 42.113 & 47.857 \\
$P$ & 0.000 & 0.000 \\
\hline
\end{tabular}

\section{DISCUSSION}

The World Health Organization defines pain as an unpleasant and emotional feeling, with or without substantial or potential tissue damage. It is one of the common causes of human suffering and the most unbearable symptom of patients with advanced cancer ${ }^{[5]}$. According to incomplete statistics, about $50 \%$ of patients with advanced tumors are accompanied by pain, and 30\% have severe and unbearable pain, which makes it become a worldwide problem ${ }^{[6]}$.

Hematological tumors are composed of leukemia, multiple myeloma, and lymphoma. In recent years, the incidence of such tumors has shown a rapid rise among younger populations because of some factors such as radiation, chemistry, viruses, genetics, $e t c^{[7]}$. Due to the rapid progress of hematological tumors, patients are often at an advanced stage when they are diagnosed, and their quality of life declines sharply. What is more serious is that long-term treatment brings relatively severe trauma to the body, making them generally accompanied by varying degrees of pain, which further weakens their quality of life ${ }^{[8]}$.

In this study, 50 cases of patients with advanced hematologic tumor in our hospital were evaluated. It was found that moderate and severe pain became the true portrayal of such patients, accounting for $94.00 \%$, including $50.00 \%$ of moderate pain and $44.00 \%$ of severe pain. Due to the existence of moderate to severe pain, patients with advanced hematological tumors generally have poor sleep quality, serious psychological problems and severely low quality of life. In response to this situation, this study gave all patients pain care. Firstly, medications were provided for patients with different pain levels to reduce the pain level felt by the patients according to the threestep analgesia recommended by the World Health Organization. According to the results of existing research, after the implementation of the three-step analgesia, the pain of $80 \%$ of cancer patients is effectively relieved, and the pain of more than $75 \%$ of advanced cancer patients is relieved ${ }^{[9]}$. The pain scores in this study were significantly lower than those before the implementation of pain care, and they were in the range of patients with advanced hematological tumor. Non-medicinal pain relief is to further distract patients with advanced hematological tumors by listening to music, watching TV programs, and participating in entertainment activities, and relieve the adverse effects of pain on their body and mind. Sleep quality and quality of life have been significantly improved 
after that. A study pointed out that psychological care is supposed to run through the pain care of patients with advanced cancer ${ }^{[10]}$. The reason is that pain will cause the patient to bear a heavy physical and mental burden, especially the advanced hematological tumors have lost the opportunity to be completely cured, and the patients inevitably have psychological barriers, even suicidal tendency, so good psychological care is of great significance to relieve the patient's mental burden. In this study, through the implementation of psychological care for patients with advanced hematological tumors, their anxiety scores and depression scores were significantly reduced, which not only helped to further eliminate the adverse effects of pain, but also improved pain tolerance with the help of diversified psychological interventions threshold, actively cooperating with treatment work. Combining the above research results, this study points out that the pain care of patients with advanced hematological tumors should have diversified characteristics, that is: an organic combination of drug analgesia, non-drug analgesia, and psychological care, starting from the patient's body and mind, not only to relieve pain, we also focus on improving the quality of sleep and quality of life to provide better medical services.

In summary, patients with advanced hematological tumor generally have moderate to severe pain. Considerate pain care can help relieve pain, improve sleep quality and mental state.

\section{REFERENCE}

[1] Zhangjie Luo. Application effect of psychological intervention combined with hospice nursing in patients with advanced hematological tumor[J]. Medical Aesthetics and Cosmetology,2019,28(13):136-137.

[2] Ruirui Wei, Yan Zhang. Explore the application effect of palliative care in improving the quality of life of patients with advanced hematological tumors[J].Continuing Medical Education, 2018,32(9):114-116.

[3] Panpan Huang, Zheyuan Le. Pain evaluation and nursing intervention effect analysis of 87 patients with advanced cancer [J]. Modern Practical Medicine. 2019,31(9):1252-1254.

[4] Lili Qin, Huajuan Weng, Haixia Xiong. The effect of nursing management intervention on the nursing effect of critically ill patients with hematological tumors[J]. Journal of Practical Medical Techniques, 2018,25(5):572-573.

[5] Xiujuan Liu, Yufeng Li, Yuyan Ma. Analysis of the effect of comfort nursing on patients with advanced hematological tumors[J]. China Health Care Nutrition, 2018,28(28):208.

[6] Huijuan Zheng. Observation on the effect of comfort nursing on patients with advanced hematological tumors[J]. Guide of China Medical, 2018,16(9): 256-257.

[7] Yanhui Du. The effect of holistic nursing on improving the negative emotion and pain of leukemia patients with chemotherapy[J]. International Journal of Nursing, 2019,38(22):3779-3780.

[8] Guihua $\mathrm{Lu}$. The application effect of physiology nursing and psychological "arousal" concept in chemotherapy of elderly patients with leukemia[J]. International Journal of Nursing, 2019,38(20):3311-3314.

[9] Hong Zhang, Ju Dong, Li Zhang, etc. Analysis of the influence of pain management on the pain and psychological state of children with tumor[J]. Anti-tumor Pharmacy, 2018,8(5):825-828.

[10] Xiaolan Zhang, Cuirong Liang. Analysis of the effect of comfort nursing on patients with advanced hematological tumors[J]. Journal of Mathematical Medicine, 2017,30(9):1382-1383. 